\title{
Robust Exponential Synchronization of a Class of Chaotic Systems with Variable Convergence Rates via the Saturation Control
}

\author{
Meichun Huang, Runzi Luo $\mathbb{D}$, Jiaojiao Fu, and Haipeng Su \\ Department of Mathematics, Nanchang University, Nanchang 330031, China \\ Correspondence should be addressed to Runzi Luo; luo_rz@163.com
}

Received 1 July 2020; Revised 24 August 2020; Accepted 25 September 2020; Published 7 October 2020

Academic Editor: Xianggui Guo

Copyright (C) 2020 Meichun Huang et al. This is an open access article distributed under the Creative Commons Attribution License, which permits unrestricted use, distribution, and reproduction in any medium, provided the original work is properly cited.

\begin{abstract}
This article is concerned with the exponential synchronization of a class of the chaotic systems with external disturbance via the saturation control. Through appropriate coordinate transformation, the exponential synchronization is translated into the asymptotic stability of the error system. By using the Lyapunov stability theory, a novel sufficient condition which possesses the exponential convergence rate $\lambda$ is presented. The rich choices of the exponential convergence rate $\lambda$ turn our scheme more general than some existing approaches. Numerical simulations are employed to the Genesio chaotic system and the Coullet chaotic system to illustrate the ability and effectiveness of the presented approach.
\end{abstract}

\section{Introduction}

Synchronization exists in many systems, such as chaotic system, complex network system, and neural network system. Since Pecora and Carroll [1] proposed the drive-respond synchronization scenario in 1990, chaos synchronization has turned out to be a hot topic. So far, many kinds of synchronization schemes have been proposed by the experts, such as the complete synchronization [1], lag synchronization [2], phase synchronization [3], projective synchronization [4], and combination synchronization [5]. Chaos synchronization, owing to its great application in engineering science, medicine, secure communication, and telecommunications, has attracted widespread concern in a variety of areas and has been studied extensively during the last decades [6-12]. However, most of the synchronization schemes are based on asymptotic stability. From a practical point of view, chaos systems are required not only to be synchronized but also with a fast synchronizing rate. Thus, the exponential synchronization, which can quantify the rate of convergence and possesses the faster convergence speed than that of general asymptotic stability, has received much attention in many research fields. For example, the study in [13] investigated the exponential synchronization of the chaotic Lur'e systems via the stochastic sampled-data controller. The authors in paper [14] discussed the exponential synchronization of a class of fractional-order chaotic systems based on the discontinuous input. The exponential synchronization of a special chaotic system which has no linear term was considered in paper [15] by using the exponential stability theorem. The study in [16] discussed the exponential synchronization of a class of fractional-order chaotic systems with uncertainty. A new criterion was proposed by using the linear matrix inequalities approach. The exponential synchronization between two identical xian chaotic systems was considered in paper [17]. By using algebraic Riccati equation, a linear feedback controller was presented.

In the literature, most of the published papers concerned the exponential synchronization (see [13-17] for example), and the convergence rate is fixed which means that the convergence speed is constant. From a practical point of view, it is to be hoped that the exponential synchronization can be achieved as soon as possible. On the other hand, it is 
well known that every physical actuator is subject to saturation. When the actuator is saturated, the performance of the designed control system will be deteriorated seriously. In order to improve the control performance, the effect of saturation should be incorporated into the design of the controlled system.

Motivated by such circumstances, in this paper, we investigate the exponential drive-response synchronization of a class of chaotic systems with plant uncertainties via the saturation control. A novel synchronization controller, in which a variable convergence rate is incorporated into the control law, is proposed. Numerical studies are provided to verify the effectiveness of the given scheme.

The remainder of this paper is organized as follows. In Section 2 and Section 3, the problem formulation and driveresponse synchronization schemes are proposed, respectively. The numerical example is provided in Section 4 to demonstrate the effectiveness and the benefit of the proposed control scheme. Finally, the concluding remarks are drawn in Section 5.

\section{Problem Formulation}

In order to observe the chaotic synchronization phenomenon, in this paper, the following system is considered as the drive system:

$$
\left\{\begin{array}{l}
\dot{x}_{1}=x_{2}, \\
\dot{x}_{2}=x_{3}, \\
\cdots \\
\dot{x}_{n-1}=x_{n}, \\
\dot{x}_{n}=f(x)+d_{m},
\end{array}\right.
$$

where $x=\left(x_{1}, x_{2}, \ldots, x_{n}\right)^{T}$ is the state variable, $f(x)$ is a continuous function, and $d_{m}$ is the external disturbance.

Based on system (1), the response system is given as

$$
\left\{\begin{array}{l}
\dot{y}_{1}=y_{2}, \\
\dot{y}_{2}=y_{3}, \\
\cdots \\
\dot{y}_{n-1}=y_{n}, \\
\dot{y}_{n}=g(y)+d_{r}+u,
\end{array}\right.
$$

where $y=\left(y_{1}, y_{2}, \ldots, y_{n}\right)^{T}$ is the state variable, $g(y)$ is a continuous function, and $d_{r}$ is the external disturbance. $u$ is the controller which is defined as

$$
u= \begin{cases}u_{0}, & v>u_{0}, \\ v, & |v| \leq u_{0}, \\ -u_{0}, & v<-u_{0},\end{cases}
$$

where $u_{0}$ is a constant which can be designed by the controller.

For the purpose of facilitating the analysis and design, the controller $u$ is represented as

$$
u=v-\phi(v)
$$

where

$$
\phi(v)= \begin{cases}v-u_{0}, & v>u_{0}, \\ 0, & |v| \leq u_{0}, \\ v+u_{0}, & v<-u_{0} .\end{cases}
$$

The error variable is defined as $e=\left(e_{1}, e_{2}, \ldots, e_{n}\right)^{T}=$ $\left(y_{1}-x_{1}, y_{2}-x_{2}, \ldots, y_{n}-x_{n}\right)^{T}$. By subtracting system (1) from system (2), the following error system is obtained:

$$
\left\{\begin{array}{l}
\dot{e}_{1}=e_{2}, \\
\dot{e}_{2}=e_{3}, \\
\cdots \\
\dot{e}_{n-1}=e_{n}, \\
\dot{e}_{n}=g(y)-f(x)+d_{r}-d_{m}+u .
\end{array}\right.
$$

Assumption 1. Suppose $d_{r}, d_{m}$, and $\phi(v)$ are all bounded which means that there is a constant $M>0$ such that

$$
\left|d_{r}-d_{m}-\phi(v)\right| \leq M
$$

where $M$ is known in advance.

Remark 1. It is well known that the chaos attractor is bounded which means that $\phi(v)$ is also bounded. In addition, the external disturbances $d_{r}$ and $d_{m}$ are bounded, and thus, Assumption 1 is reasonable.

Definition 1. System (1) and system (2) are said to be globally exponentially synchronized if there exist constants $\alpha(>0)$ and $\lambda(>0)$ such that $\left|e_{i}\right| \leq \alpha e^{-\lambda t}$ hold for any initial values and $t \geq 0$, where $\lambda$ is called as the exponential convergence rate, $i=1,2, \ldots, n$.

\section{Synchronization Schemes}

Lemma 1. (see [18]). Suppose $\dot{x}=-\delta x+\phi(t)$. If $\delta>0$ and $\lim _{t \rightarrow \infty} \phi(t)=0$, then $\lim _{t \rightarrow \infty} x(t)=0$, where $x \in R$ is the state variable and $\phi(t)$ is a continuous function.

Now, we introduce the new variable $z_{i}$ which is expressed as

$$
z_{i}=e^{\lambda t} e_{i}, \quad i=1,2, \ldots, n .
$$

According to system (6), one obtains

$$
\dot{z}_{i}=\lambda e^{\lambda t} e_{i}+e^{\lambda t} \dot{e}_{i}, \quad i=1,2, \ldots, n-1 .
$$

Thus, system (6) can be converted into the following system (10): 


$$
\left\{\begin{array}{l}
\dot{z}_{1}=\lambda z_{1}+z_{2}, \\
\dot{z}_{2}=\lambda z_{2}+z_{3}, \\
\vdots \quad \quad \quad \\
\dot{z}_{n-1}=\lambda z_{n-1}+z_{n}, \\
\dot{z}_{n}=\lambda z_{n}+e^{\lambda t}\left(g(y)-f(x)+d_{r}-d_{m}+v-\phi(v)\right) .
\end{array}\right.
$$

Theorem 1. If $\lim _{t \rightarrow \infty} z_{i}=0$, then there exists $\alpha>0$ and $\lambda>0$ such that $\left|e_{i}\right| \leq \alpha e^{-\lambda t}$ which means that system (1) and system (2) can reach exponential synchronization, where $i=1,2, \cdots, n$.

Proof. If $\lim _{t \longrightarrow \infty} z_{i}(t)=0$, then for any $\epsilon>0$, there exists time $T>0$ such that for $t>T$, we have $\left|z_{i}\right| \leq \varepsilon$. Since the state variables of chaotic systems are bounded, therefore for $t \in[0, T]$, there exists $\epsilon_{1}>0$ such that $\left|z_{i}\right| \leq \varepsilon_{1}$. Let $\alpha=\max \left\{\varepsilon_{1}, \varepsilon\right\}$; then, for any $t \geq 0$, we obtain $\left|z_{i}\right| \leq \alpha$. Note that

$$
\left|e_{i}\right|=e^{-\lambda t}\left|z_{i}\right| \leq \alpha e^{-\lambda t} .
$$

Thus, based on Definition 1, we conclude that system (1) and system (2) can reach exponential synchronization.
Theorem 2. If $\lim _{t \rightarrow \infty} z_{1}=0$, then $\lim _{t \rightarrow \infty} z_{i}=0$ which means that system (1) and system (2) can reach exponential synchronization, where $i=1,2, \ldots, n$.

Proof. Suppose $\lim _{t \longrightarrow \infty} z_{1}=0$, then $\lim _{t \longrightarrow \infty} \dot{z}_{1}(t)=0$. In view of that

$$
\dot{z}_{1}=\lambda z_{1}+z_{2}
$$

we have

$$
\lim _{t \longrightarrow \infty} \dot{z}_{1}=\lim _{t \longrightarrow \infty} \lambda z_{1}+\lim _{t \longrightarrow \infty} z_{2}=0,
$$

which implies that

$$
\lim _{t \longrightarrow \infty} z_{2}=0 .
$$

In the same way, we can obtain $\lim _{t \longrightarrow \infty} z_{i}=0, i=3,4, \ldots, n$. According to Theorem 1, we know that system (1) and system (2) can reach exponential synchronization.

\section{Theorem 3. If}

$$
\left\{\begin{array}{l}
v=f(x)-g(y)-\lambda \zeta-\xi-M \operatorname{sign}\left(e^{\lambda t} \zeta\right), \\
\xi=C_{n}^{0}(\lambda+\beta)^{n} e_{1}+C_{n}^{1}(\lambda+\beta)^{n-1} e_{2}+C_{n}^{2}(\lambda+\beta)^{n-2} e_{3}+\cdots+C_{n}^{n-1}(\lambda+\beta) e_{n}, \\
\zeta=C_{n-1}^{0}(\lambda+\beta)^{n-1} e_{1}+C_{n-1}^{1}(\lambda+\beta)^{n-2} e_{2}+C_{n-1}^{2}(\lambda+\beta)^{n-3} e_{3}+\cdots+C_{n-1}^{n-1} e_{n},
\end{array}\right.
$$

then system (1) and system (2) can reach exponential synchronization, where $\beta>0$.

Proof. Based on Theorem 2, we know that if

$$
\lim _{t \longrightarrow \infty} z_{1}=0,
$$

then system (1) and system (2) can reach exponential synchronization. In order to obtain

$$
\lim _{t \longrightarrow \infty} z_{1}=0,
$$

in the first step, we choose the Lyapnov function $V_{1}$ as

$$
V_{1}=\frac{1}{2} z_{1}^{2} \text {. }
$$

Its derivative is

$$
\begin{aligned}
\dot{V}_{1} & =z_{1} \dot{z}_{1}=z_{1}\left(\lambda z_{1}+z_{2}\right)=-\beta z_{1}^{2}+z_{1}\left((\lambda+\beta) z_{1}+z_{2}\right) \\
& =-\beta z_{1}^{2}+z_{1}\left(C_{1}^{0}(\lambda+\beta) z_{1}+C_{1}^{1} z_{2}\right) .
\end{aligned}
$$

Let us suppose that $\lim _{t \rightarrow \infty}\left(C_{1}^{0}(\lambda+\beta) z_{1}+C_{1}^{1} z_{2}\right)=0$. In this case, we can show that $z_{1}$ is bounded. In fact, If $z_{1}$ is unbounded, i.e., $\lim _{t \longrightarrow \infty} z_{1}=\infty$. Then, there exists a finite time $T$, such that when $t>T$, we have

$$
\dot{V}_{1}=-\beta z_{1}^{2}+z_{1}\left(C_{1}^{0}(\lambda+\beta) z_{1}+C_{1}^{1} z_{2}\right)<0 .
$$

In light of the Lyapunov stability theory, we obtain $\lim _{t \rightarrow \infty} z_{1}=0$ which is contradicted with $\lim _{t \rightarrow \infty} z_{1}=\infty$. Therefore, $z_{1}$ is bounded; then, $\lim _{t \longrightarrow \infty} z_{1}\left(C_{1}^{0}(\lambda+\beta) z_{1}+\right.$ $\left.C_{1}^{1} z_{2}\right)=0$. According to Lemma 1, we know that $\lim _{t \rightarrow \infty} z_{1}=0$.

Based on the above analysis, one can derive that if $\lim _{t \rightarrow \infty}\left(C_{1}^{0}(\lambda+\beta) z_{1}+C_{1}^{1} z_{2}\right)=0$, then $\lim _{t \rightarrow \infty} z_{1}=0$. Now, in order to obtain $\lim _{t \rightarrow \infty}\left(C_{1}^{0}(\lambda+\beta) z_{1}+C_{1}^{1} z_{2}\right)=0$, in the second step, we choose the Lyapnov function $V_{2}$ as

$$
V_{2}=\frac{1}{2}\left(C_{1}^{0}(\lambda+\beta) z_{1}+C_{1}^{1} z_{2}\right)^{2} .
$$

The derivative of $V_{2}$ is

$$
\begin{aligned}
\dot{V}_{2}= & \left((\lambda+\beta) z_{1}+z_{2}\right)\left((\lambda+\beta) \dot{z}_{1}+\dot{z}_{2}\right) \\
= & \left((\lambda+\beta) z_{1}+z_{2}\right)\left((\lambda+\beta)\left(\lambda z_{1}+z_{2}\right)+\lambda z_{2}+z_{3}\right) \\
= & -\beta\left((\lambda+\beta) z_{1}+z_{2}\right)^{2}+\left((\lambda+\beta) z_{1}+z_{2}\right) \\
& \cdot\left(C_{2}^{0}(\lambda+\beta)^{2} z_{1}+C_{2}^{1}(\lambda+\beta) z_{2}+C_{2}^{2} z_{3}\right) .
\end{aligned}
$$


Similarly, according to Lemma 1 , if

$$
\lim _{t \longrightarrow \infty}\left(C_{2}^{0}(\lambda+\beta)^{2} z_{1}+C_{2}^{1}(\lambda+\beta) z_{2}+C_{2}^{2} z_{3}\right)=0,
$$

then

$$
\lim _{t \longrightarrow \infty}\left(C_{1}^{0}(\lambda+\beta) z_{1}+C_{1}^{1} z_{2}\right)=0 .
$$

In view of step 1 , we have

$$
\lim _{t \rightarrow \infty} z_{1}=0 .
$$

Suppose that in the $i$ th step, we have proven that if $\lim _{t \rightarrow \infty} \rho=0$, then $\lim _{t \rightarrow \infty} z_{1}=0$, where

$$
\begin{aligned}
\rho= & C_{i}^{0}(\lambda+\beta)^{i} z_{1}+C_{i}^{1}(\lambda+\beta)^{i-1} z_{2}+C_{i}^{2}(\lambda+\beta)^{i-2} z_{3} \\
& +C_{i}^{3}(\lambda+\beta)^{i-3} z_{4}+\cdots+C_{i}^{i-1}(\lambda+\beta) z_{i}+C_{i}^{i} z_{i+1} .
\end{aligned}
$$

Taking

$$
V_{i+1}=\frac{1}{2} \rho^{2},
$$

then we have $\dot{V}_{i+1}=\rho \dot{\rho}=-\beta \rho^{2}+\rho(\dot{\rho}+\beta \rho)$.

Thus, we have

$$
\begin{aligned}
\dot{V}_{i+1}= & -\beta \rho^{2}+\rho\left(C_{i}^{0}(\lambda+\beta)^{i}\left(\lambda z_{1}+z_{2}\right)+C_{i}^{1}(\lambda+\beta)^{i-1}\right. \\
& \cdot\left(\lambda z_{2}+z_{3}\right)+C_{i}^{2}(\lambda+\beta)^{i-2}\left(\lambda z_{3}+z_{4}\right) \\
& +C_{i}^{3}(\lambda+\beta)^{i-3}\left(\lambda z_{4}+z_{5}\right)+\cdots+C_{i}^{i-1}(\lambda+\beta) \\
& \cdot\left(\lambda z_{i}+z_{i+1}\right)+C_{i}^{i}\left(\lambda z_{i+1}+z_{i+2}\right) \\
& +\beta\left(C_{i}^{0}(\lambda+\beta)^{i} z_{1}+C_{i}^{1}(\lambda+\beta)^{i-1} z_{2}+C_{i}^{2}(\lambda+\beta)^{i-2} z_{3}\right. \\
& \left.\left.+C_{i}^{3}(\lambda+\beta)^{i-3} z_{4}+\cdots+C_{i}^{i-1}(\lambda+\beta) z_{i}+C_{i}^{i} z_{i+1}\right)\right) \\
= & -\beta \rho^{2}+\rho\left(C_{i}^{0}(\lambda+\beta)^{i+1} z_{1}+(\lambda+\beta)^{i}\left(C_{i}^{0}+C_{i}^{1}\right) z_{2}\right. \\
& +(\lambda+\beta)^{i-1}\left(C_{i}^{1}+C_{i}^{2}\right) z_{3} \\
& \left.+\cdots+(\lambda+\beta)\left(C_{i}^{i-1}+C_{i}^{i}\right) z_{i+1}+C_{i}^{i} z_{i+2}\right) .
\end{aligned}
$$

Using the mathematical formula

$$
C_{n}^{m}+C_{n}^{m-1}=C_{n+1}^{m},
$$

we obtain

$$
\begin{aligned}
\dot{V}_{i+1}= & -\beta \rho^{2}+\rho\left(C_{i+1}^{0}(\lambda+\beta)^{i+1} z_{1}+C_{i+1}^{1}(\lambda+\beta)^{i} z_{2}\right. \\
& +C_{i+1}^{2}(\lambda+\beta)^{i-1} z_{3} \\
& \left.+\cdots+C_{i+1}^{i}(\lambda+\beta) z_{i+1}+C_{i+1}^{i+1} z_{i+2}\right), \quad i \leq n-2 .
\end{aligned}
$$

Then, by Lemma 1 , we know that if

$$
\begin{aligned}
& \lim _{t \longrightarrow \infty}\left(C_{i+1}^{0}(\lambda+\beta)^{i+1} z_{1}+C_{i+1}^{1}(\lambda+\beta)^{i} z_{2}+C_{i+1}^{2}(\lambda+\beta)^{i-1} z_{3}\right. \\
& \left.\quad+\cdots+C_{i+1}^{i}(\lambda+\beta) z_{i+1}+C_{i+1}^{i+1} z_{i+2}\right)=0,
\end{aligned}
$$

then

$$
\lim _{t \rightarrow \infty} z_{1}=0
$$

In the above equation, if we set $i=n-2$, then one can conclude that

$$
\lim _{t \longrightarrow \infty} \rho_{1}=0
$$

which implies that

$$
\lim _{t \rightarrow \infty} z_{1}=0
$$

where

$$
\begin{aligned}
\rho_{1}= & C_{n-1}^{0}(\lambda+\beta)^{n-1} z_{1}+C_{n-1}^{1}(\lambda+\beta)^{n-2} z_{2} \\
& +C_{n-1}^{2}(\lambda+\beta)^{n-3} z_{3}+\cdots+C_{n-1}^{n-2}(\lambda+\beta) z_{n-1}+C_{n-1}^{n-1} z_{n} .
\end{aligned}
$$

The derivative of $\rho_{1}$ is

$$
\begin{aligned}
\dot{\rho}_{1}= & C_{n-1}^{0}(\lambda+\beta)^{n-1}\left(\lambda z_{1}+z_{2}\right)+C_{n-1}^{1}(\lambda+\beta)^{n-2}\left(\lambda z_{2}+z_{3}\right) \\
& +C_{n-1}^{2}(\lambda+\beta)^{n-3}\left(\lambda z_{3}+z_{4}\right)+\cdots+C_{n-1}^{n-2}(\lambda+\beta)\left(\lambda z_{n-1}+z_{n}\right) \\
& +C_{n-1}^{n-1}\left(\lambda z_{n}+e^{\lambda t}\left(g(y)-f(x)+d_{r}-d_{m}-\phi(v)+v\right)\right) .
\end{aligned}
$$

Choose the following Lyapunov function:

$$
V_{n}=\frac{1}{2} \rho_{1}^{2} \text {. }
$$

The derivative of $V_{n}$ is

$$
\dot{V}_{n}=\rho_{1} \dot{\rho}_{1}=-\beta \rho_{1}^{2}+\rho_{1}\left(\dot{\rho}_{1}+\beta \rho_{1}\right) \text {. }
$$

Thus, we have

$$
\begin{aligned}
\dot{V}_{n}= & -\beta \rho_{1}^{2}+\rho_{1}\left(C_{n-1}^{0}(\lambda+\beta)^{n-1}\left(\lambda z_{1}+z_{2}\right)+C_{n-1}^{1}(\lambda+\beta)^{n-2}\left(\lambda z_{2}+z_{3}\right)\right. \\
& +C_{n-1}^{2}(\lambda+\beta)^{n-3}\left(\lambda z_{3}+z_{4}\right)+\cdots+C_{n-1}^{n-2}(\lambda+\beta)\left(\lambda z_{n-1}+z_{n}\right) \\
& +C_{n-1}^{n-1}\left(\lambda z_{n}+e^{\lambda t}\left(g(y)-f(x)+d_{r}-d_{m}-\phi(v)+v\right)\right) \\
& +\beta\left(C_{n-1}^{0}(\lambda+\beta)^{n-1} z_{1}+C_{n-1}^{1}(\lambda+\beta)^{n-2} z_{2}+C_{n-1}^{2}(\lambda+\beta)^{n-3} z_{3} .\right.
\end{aligned}
$$

Thus, we have 


$$
\begin{aligned}
\dot{V}_{n}= & -\beta \rho_{1}^{2}+\rho_{1}\left(C_{n-1}^{0}(\lambda+\beta)^{n-1}\left(\lambda z_{1}+z_{2}\right)+C_{n-1}^{1}(\lambda+\beta)^{n-2}\left(\lambda z_{2}+z_{3}\right)\right. \\
& +C_{n-1}^{2}(\lambda+\beta)^{n-3}\left(\lambda z_{3}+z_{4}\right)+\cdots+C_{n-1}^{n-2}(\lambda+\beta)\left(\lambda z_{n-1}+z_{n}\right) \\
& +C_{n-1}^{n-1}\left(\lambda z_{n}+e^{\lambda t}\left(g(y)-f(x)+d_{r}-d_{m}-\phi(v)+v\right)\right) \\
& +\beta\left(C_{n-1}^{0}(\lambda+\beta)^{n-1} z_{1}+C_{n-1}^{1}(\lambda+\beta)^{n-2} z_{2}+C_{n-1}^{2}(\lambda+\beta)^{n-3} z_{3}\right. \\
& \left.+\cdots+C_{n-1}^{n-2}(\lambda+\beta) z_{n-1}+C_{n-1}^{n-1} z_{n}\right) \\
= & -\beta \rho_{1}^{2}+\rho_{1}\left(C_{n}^{0}(\lambda+\beta)^{n} z_{1}+C_{n}^{1}(\lambda+\beta)^{n-1} z_{2}+C_{n}^{2}(\lambda+\beta)^{n-2} z_{3}\right. \\
& +\cdots+C_{n}^{n-2}(\lambda+\beta)^{2} z_{n-1}+C_{n}^{n-1}(\lambda+\beta) z_{n} \\
& \left.+C_{n}^{n} e^{\lambda t}\left(g(y)-f(x)+d_{r}-d_{m}-\phi(v)+v\right)\right) .
\end{aligned}
$$

Note that

$$
\begin{aligned}
e^{\lambda t} \xi= & C_{n}^{0}(\lambda+\beta)^{n} z_{1}+C_{n}^{1}(\lambda+\beta)^{n-1} z_{2}+C_{n}^{2}(\lambda+\beta)^{n-2} z_{3} \\
& +\cdots+C_{n}^{n-2}(\lambda+\beta)^{2} z_{n-1}+C_{n}^{n-1}(\lambda+\beta) z_{n}, \\
e^{\lambda t} \zeta= & \rho_{1},
\end{aligned}
$$

$$
\begin{aligned}
& \rho_{1} e^{\lambda t}\left(g(y)-f(x)+d_{r}-d_{m}-\phi(v)+v\right) \\
& \quad=\rho_{1} e^{\lambda t}\left(d_{r}-d_{m}-\phi(v)-\lambda \zeta-\xi-M \operatorname{sign}\left(e^{\lambda t} \zeta\right)\right) \\
& \leq-\rho_{1} e^{\lambda t} \xi-\lambda \rho_{1}^{2}+e^{\lambda t}\left|\rho_{1}\right| M-e^{\lambda t} \rho_{1} M \operatorname{sign}\left(e^{\lambda t} \zeta\right) \\
& \quad \leq-\rho_{1} e^{\lambda t} \xi-\lambda \rho_{1}^{2} .
\end{aligned}
$$

By substituting (15), (41), and (42) into (40), one has

$$
\dot{V}_{n} \leq-(\lambda+\beta) \rho_{1}^{2} \text {. }
$$

According to Lyapunov's stability theory, we obtain

$$
\lim _{t \rightarrow \infty} \rho_{1}=0 .
$$

Therefore, $\lim _{t \rightarrow \infty} z_{1}=0$. According to Theorem 1, we know that system (1) and system (2) can reach exponential synchronization.

Remark 2. From Theorem 1, one can see that the convergence rate of system (6) is $\lambda$. Since $\lambda$ is variable and can be chosen freely by the controller, Theorem 3 ensures that system (1) and system (2) can reach exponential synchronization with variable convergence rates via the saturation control.

Remark 3. The convergence rate of many published papers [13-17] concerned that the exponential synchronization is fixed which means that the convergence speed is constant. However, from Theorem 3, it is easy to see that the convergence rate of the exponential synchronization is variable. In addition, the control schemes proposed in papers [13-17] have not taken into consideration the effect of saturation. Note that in reality, the physical actuator is usually subject to saturation; therefore, our control strategy is applicable to the practical systems.
In view of that most of the chaotic systems are 3- or 4-dimension systems, now we discuss two special cases.

Case 1. The chaotic system is a 3-dimension system, i.e., $n=3$. Based on Theorem 2, in this case, we obtain

$$
\begin{aligned}
v= & -g(y)+f(x)-\left((\lambda+\beta)^{3} e_{1}+3(\lambda+\beta)^{2} e_{2}+3(\lambda+\beta) e_{3}\right) \\
& \left.-\lambda\left((\lambda+\beta)^{2} e_{1}+2(\lambda+\beta) e_{2}+e_{3}\right)\right) \\
& -M \operatorname{sign}\left(e^{\lambda t}\left((\lambda+\beta)^{2} e_{1}+2(\lambda+\beta) e_{2}+e_{3}\right)\right) .
\end{aligned}
$$

Case 2. The chaotic system is a 4-dimension system, i.e., $n=4$. Based on Theorem 2, in this case, we have

$$
\begin{aligned}
v= & -g(y)+f(x)-\left((\lambda+\beta)^{4} e_{1}+4(\lambda+\beta)^{3} e_{2}+6(\lambda+\beta)^{2} e_{3}\right. \\
& \left.+4(\lambda+\beta) e_{4}\right) \\
& -\lambda\left((\lambda+\beta)^{3} e_{1}+3(\lambda+\beta)^{2} e_{2}+3(\lambda+\beta) e_{3}+e_{4}\right) \\
& -M \operatorname{sign}\left(e^{\lambda t}\left((\lambda+\beta)^{3} e_{1}+3(\lambda+\beta)^{2} e_{2}+3(\lambda+\beta) e_{3}+e_{4}\right)\right) .
\end{aligned}
$$

\section{Numerical Simulations}

In the sequel, the Genesio chaotic system and the Coullet chaotic system are used to test the effectiveness of the proposed method.

The Genesio chaotic system, proposed by Genesio and Tesi [19], is given as

$$
\left\{\begin{array}{l}
\dot{x}_{1}=x_{2}, \\
\dot{x}_{2}=x_{3}, \\
\dot{x}_{3}=-a_{1} x_{1}-b_{1} x_{2}-c_{1} x_{3}+x_{1}^{2}+d_{m},
\end{array}\right.
$$

where $x_{1}, x_{2}$, and $x_{3}$ are state variables and $a_{1}, b_{1}$, and $c_{1}\left(c_{1} b_{1}<a_{1}\right)$ are the positive real parameters. $d_{m}$ is the external disturbance. When $d_{m}=0$, system (47) is chaotic and the chaos attractor is shown in Figure 1 with $c_{1}=1.2, b_{1}=2.92$, and $a_{1}=6$.

In the synchronization scheme, we suppose that system (47) is the drive system and the Coullet system [20] is the response system which is described by 


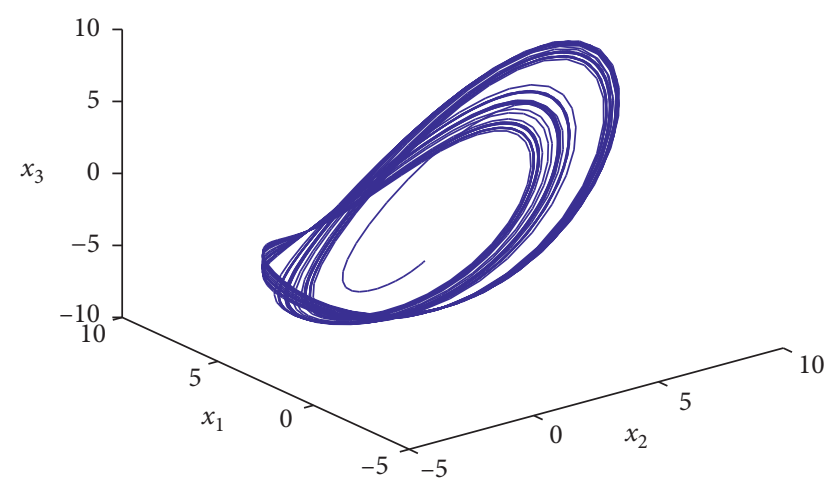

Figure 1: The chaos attractor of system (47) with $x_{1}(0)=2, x_{2}(0)=1$, and $x_{3}(0)=-4$.

$$
\left\{\begin{array}{l}
\dot{y}_{1}=y_{2}, \\
\dot{y}_{2}=y_{3}, \\
\dot{y}_{3}=a_{2} y_{1}-b_{2} y_{2}-c_{2} y_{3}-y_{1}^{3}+d_{r}+u,
\end{array}\right.
$$

where $y_{1}, y_{2}$, and $y_{3}$ are state variables and $a_{2}, b_{2}$, and $c_{2}$ are positive constants. $d_{r}$ is the external disturbance, and $u$ is the controller. When $d_{r}=0$ and $u=0$ and $a_{2}=5.5, b_{2}=3.5$, and $c_{2}=1.0$, system (48) is chaotic and the chaos attractor is depicted in Figure 2.

Based on (45), the $v$ can be chosen as

$$
\begin{aligned}
v= & -\left(a_{2} y_{1}-b_{2} y_{2}-c_{2} y_{3}-y_{1}^{3}\right)+\left(-a_{1} x_{1}-b_{1} x_{2}-c_{1} x_{3}+x_{1}^{2}\right) \\
& -\left((\lambda+\beta)^{3} e_{1}+3(\lambda+\beta)^{2} e_{2}+3(\lambda+\beta) e_{3}\right) \\
& \left.-\lambda\left((\lambda+t \beta)^{2} e_{1} t+n 2 q(\lambda+\beta) h e_{2+} x e_{3}\right)\right) \\
& -M \operatorname{sign}\left(e^{\lambda t}\left((\lambda+\beta)^{2} e_{1}+2(\lambda+\beta) e_{2}+e_{3}\right)\right),
\end{aligned}
$$

where $e_{1}=y_{1}-x_{1}, e_{2}=y_{2}-x_{2}$, and $e_{3}=y_{3}-x_{3}$. According to Theorem 3 , the exponential synchronization between system (47) and system (48) will be reached.

In the simulation process, we set $c_{1}=1.2, b_{1}=2.92$, and $a_{1}=6$ and $a_{2}=5.5, b_{2}=3.5$, and $c_{2}=1$ such that the two systems are chaotic. In addition, for simplicity, we take $\beta=1, d_{r}=y_{1}^{2}+0.2 \cos (t)$, and $d_{m}=x_{1}+0.2 \sin (t)$. The $M$ and $u_{0}$ are selected as $M=30$ and $u_{0}=30$. The initial conditions of the drive and response systems are chosen as $\left(x_{1}(0), x_{2}(0), x_{3}(0)\right)=(2,1,-4)$ and $\left(y_{1}(0), y_{2}(0), y_{3}(0)\right)=(4,-1,1)$, respectively.

The simulation graphs with $\lambda=1$ and $\lambda=3$ are depicted in Figures 3-7.

The time response of synchronization error variables $e_{1}, e_{2}$, and $e_{3}$ are shown in Figures $3-5$, respectively. The time response of input signal $u$ with $\lambda=1$ and $\lambda=3$ are exhibited in Figures 6 and 7, respectively. From Figures 3-5, one can easily see that the synchronization between systems (47) and (48) is realized. Meanwhile, one can also observe from Figures 3-5 that the synchronization speed of $\lambda=3$ is faster than that of $\lambda=1$.

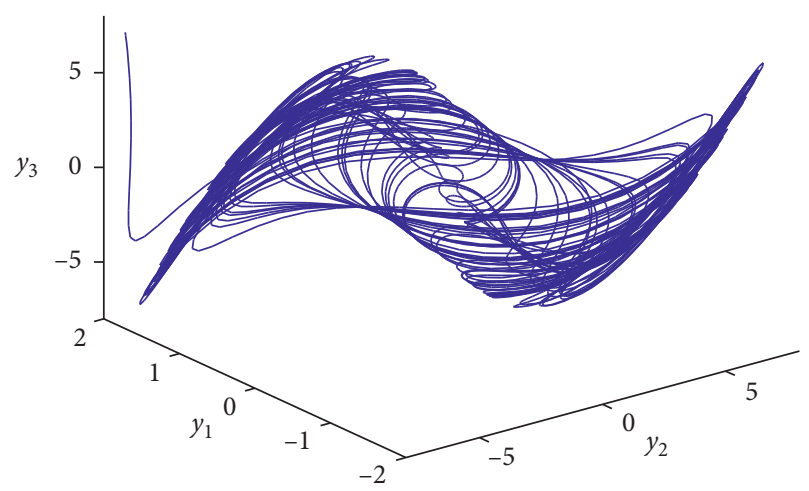

Figure 2: The chaos attractor of system (48) with $y_{1}(0)=4, y_{2}(0)=-1$, and $y_{3}(0)=1$.

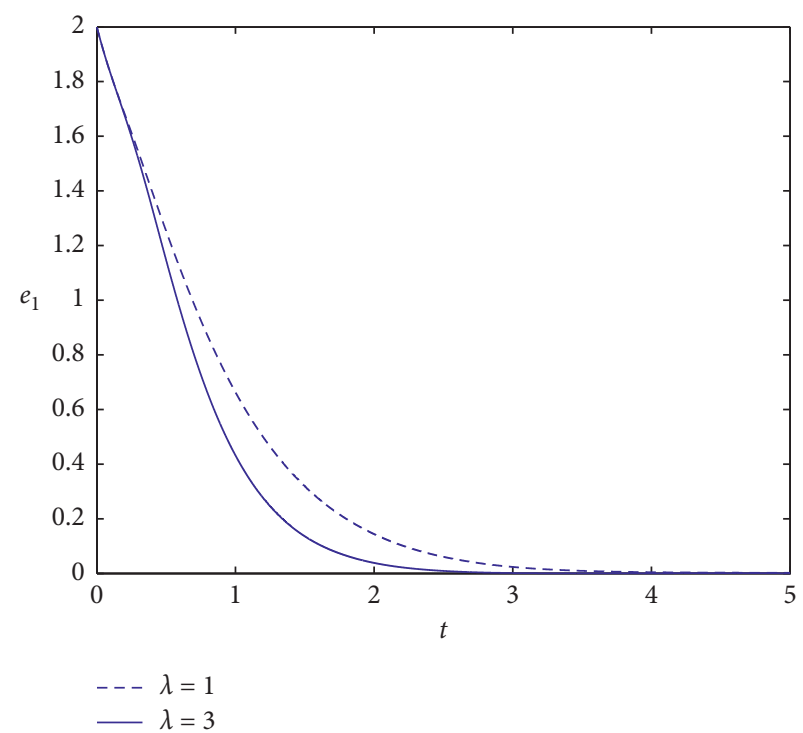

FIGURE 3: The time response of synchronization error variable $e_{1}$.

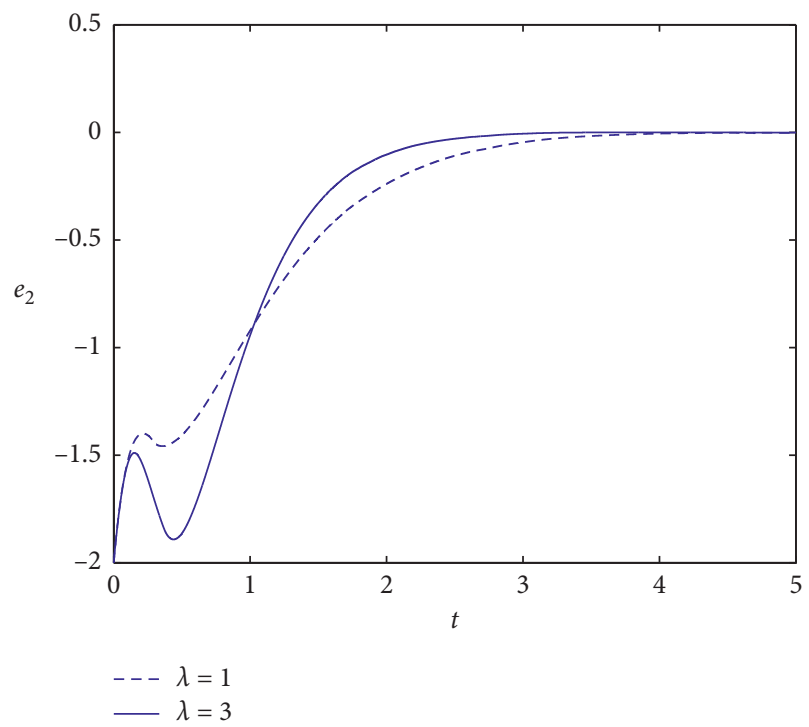

FIGURE 4: The time response of synchronization error variable $e_{2}$. 




Figure 5: The time response of synchronization error variable $e_{3}$.

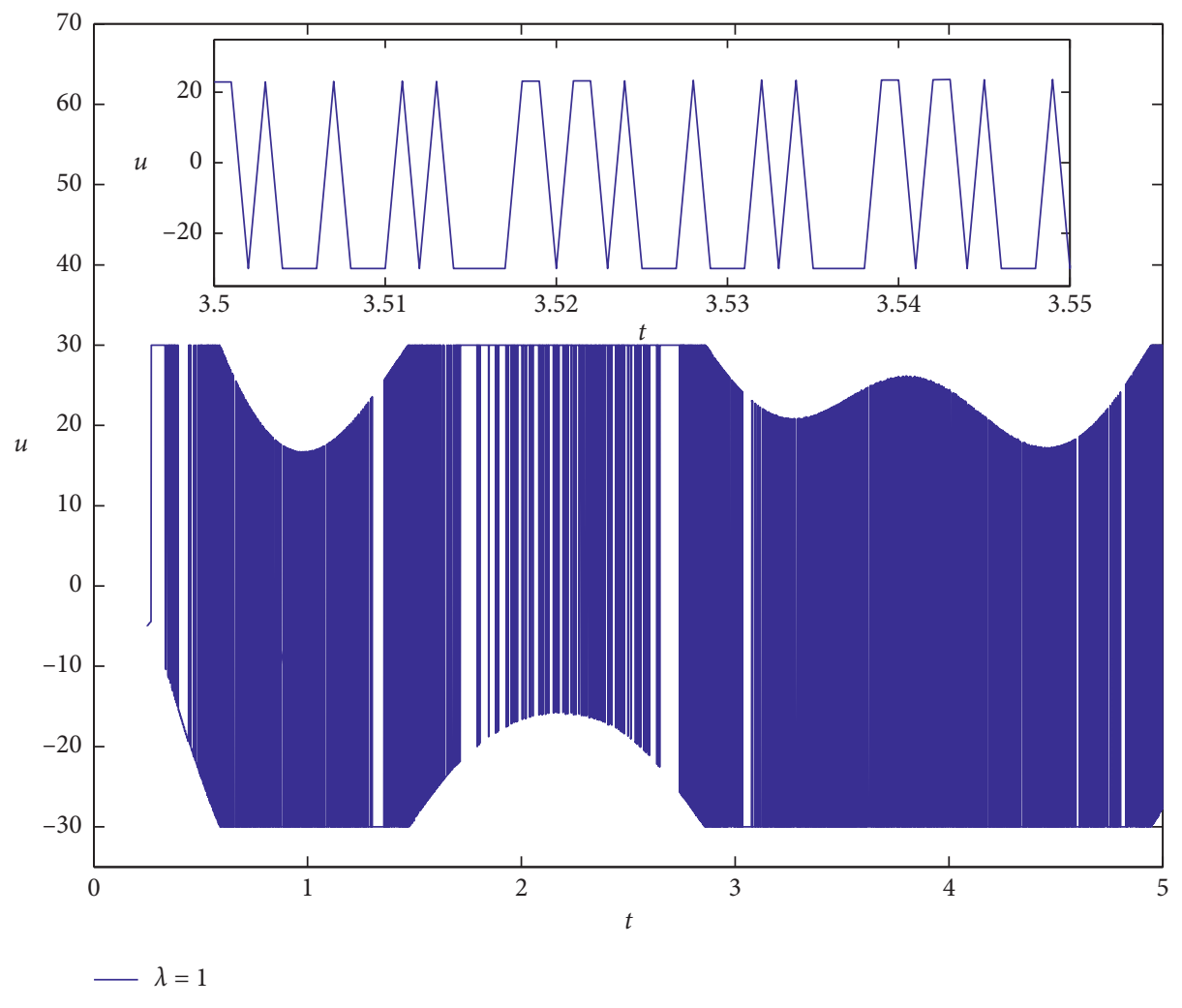

Figure 6: The time response of input signal $u$ with $\lambda=1$. 


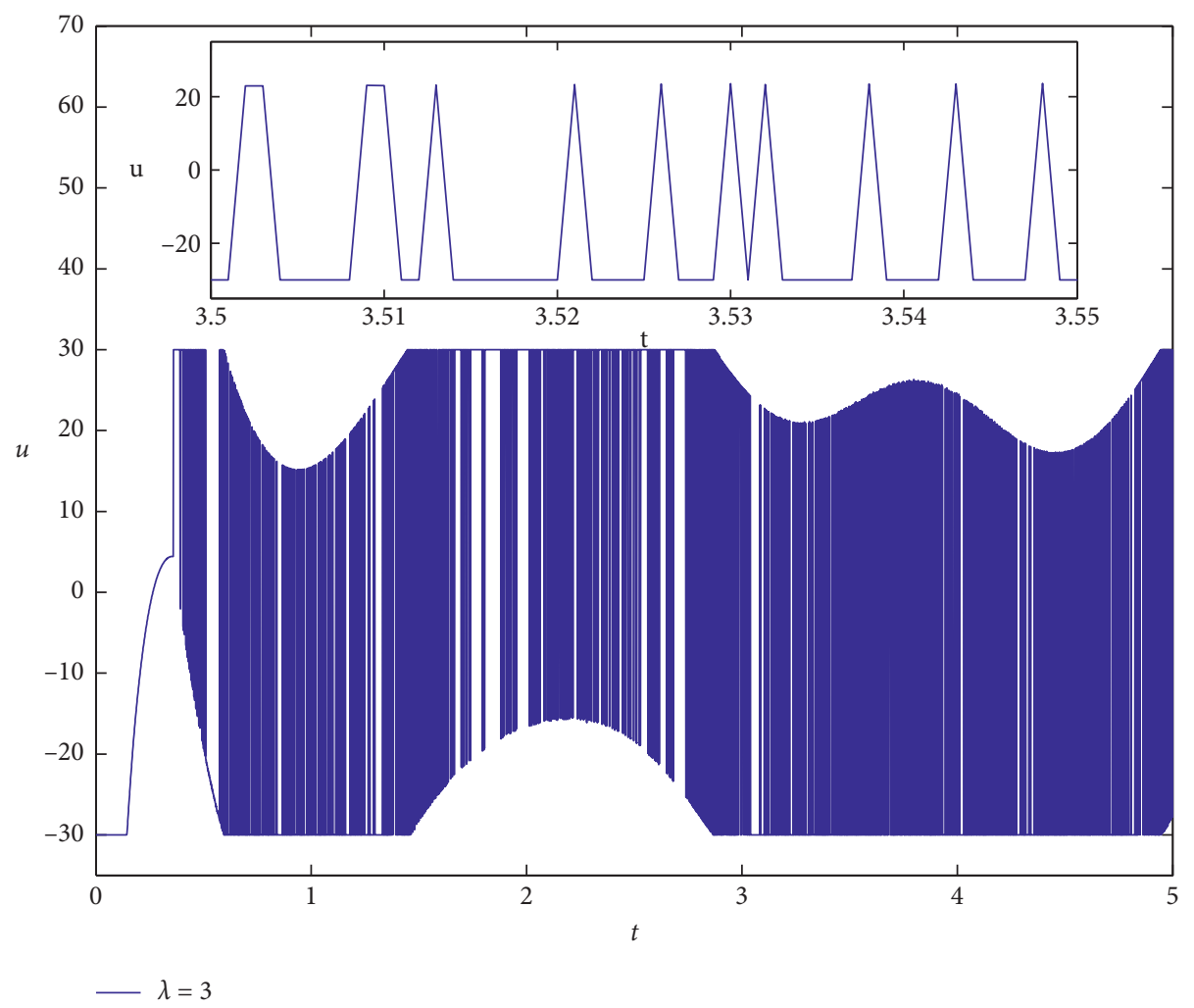

Figure 7: The time response of input signal $u$ with $\lambda=3$.

\section{Conclusions}

In this paper, the exponential synchronization of a class of $n D$ chaotic systems with external disturbances has been investigated via the coordinates transformation method. Based on the Lyapunov stability theory, a new saturation controller is presented to ensure that the coupled chaotic systems can achieve synchronization exponentially. The proposed controller contains the convergence rate $\lambda$ which can be used to control the convergence speed of the synchronization. By selecting different values of $\lambda$, the exponential synchronization will be reached with any prespecified exponential convergence rate. Numerical examples are proposed to demonstrate the usefulness and merits of our presented scheme.

\section{Data Availability}

The data used to support the findings of this study are available from the corresponding author upon request.

\section{Conflicts of Interest}

The authors declare that they have no conflicts of interest.

\section{Acknowledgments}

This work was jointly supported by the National Natural Science Foundation of China under Grant nos. 11761050 and 11361043.

\section{References}

[1] B. Yaghooti, A. Siahi Shadbad, and H. Salarieh, "Adaptive synchronization of uncertain fractional-order chaotic systems using sliding mode control techniques," Proceedings of the Institution of Mechanical Engineers, Part I: Journal of Systems and Control Engineering, vol. 234, no. 1, pp. 3-9, 2019.

[2] W. Yu and J. Cao, "Adaptive synchronization and lag synchronization of uncertain dynamical system with time delay based on parameter identification," Physica A: Statistical Mechanics and Its Applications, vol. 375, no. 2, pp. 467-482, 2007.

[3] Z. Wang and K. M. Newell, "Phase synchronization of foot dynamics in quiet standing," Neuroscience Letters, vol. 507, no. 1, pp. 47-51, 2012.

[4] F. Farivar, M. A. Shoorehdeli, M. A. Nekoui, and M. Teshnehlab, "Generalized projective synchronization for chaotic systems via Gaussian radial basis adaptive backstepping control," Chaos, Solitons \& Fractals, vol. 42, no. 2, pp. 826-839, 2009.

[5] R. Z. Luo, Y. L. Wang, and S. C. Deng, "Combination synchronization of three classic chaotic systems using active backstepping design,” Chaos, vol. 21, no. 4, 2011.

[6] L. M. Pecora and T. L. Carroll, "Synchronization of chaotic systems," Chaos, vol. 25, no. 9, 2015.

[7] B. Quan, C. Wang, J. Sun, and Y. Zhao, "A novel adaptive active control projective synchronization of chaotic systems," ASME Journal of Computational and Nonlinear Dynamics, vol. 13, no. 5, 2018.

[8] P. Balasubramaniam and P. Muthukumar, "Synchronization of chaotic systems using feedback controller: an application to Diffie-Hellman key exchange protocol and ElGamal public 
key cryptosystem," Journal of the Egyptian Mathematical Society, vol. 22, no. 3, pp. 365-372, 2014.

[9] M. Behzad, H. Salarieh, and A. Alasty, "Chaos synchronization in noisy environment using nonlinear filtering and sliding mode control," Chaos, Solitons \& Fractals, vol. 36, no. 5, pp. 1295-1304, 2008.

[10] H. Salarieh and A. Alasty, "Chaos synchronization of nonlinear gyros in presence of stochastic excitation via sliding mode control," Journal of Sound and Vibration, vol. 313, no. 3-5, pp. 760-771, 2008.

[11] K. k. Sun, L. Liu, J. B. Qiu, and G. Feng, "Fuzzy adaptive finitetime fault-tolerant control for strict-feedback nonlinear systems," IEEE Transactions on Fuzzy Systems, 2020.

[12] K. k. Sun, J. B. Qiu, H. R. Karimi, and Y. L. Fu, "Eventtriggereds robust fuzzy adaptive finite-time control of nonlinear systems with prescribed performance," IEEE Transactions on Fuzzy Systems, 2020.

[13] R. Zhang, D. Zeng, S. Zhong, K. Shi, and J. Cui, "New approach on designing stochastic sampled-data controller for exponential synchronization of chaotic Lur'e systems," Nonlinear Analysis: Hybrid Systems, vol. 29, pp. 303-321, 2018.

[14] H. Su, R. Luo, and Y. Zeng, “The exponential synchronization of a class of fractional-order chaotic systems with discontinuous input," Optik, vol. 131, pp. 850-861, 2017.

[15] B. Naderi and H. Kheiri, "Exponential synchronization of chaotic system and application in secure communication," Optik, vol. 127, no. 5, pp. 2407-2412, 2016.

[16] K. Mathiyalagan, J. H. Park, and R. Sakthivel, "Exponential synchronization for fractional-order chaotic systems with mixed uncertainties," Complexity, vol. 21, no. 1, pp. 114-125, 2015.

[17] J. Humberto Pérez-Cruz, P. A. Tamayo-Meza, M. Figueroa et al., "Exponential synchronization of chaotic Xian system using linear feedback control," Complexity, vol. 2019, Article ID 4706491, 10 pages, 2019.

[18] R. Z. Luo and L. M. He, "PC synchronization of a class of chaotic systems via event triggered control," Chinese Physics $B$, vol. 23, 2014.

[19] R. Genesio and A. Tesi, "Harmonic balance methods for the analysis of chaotic dynamics in nonlinear systems," Automatica, vol. 28, no. 3, pp. 531-548, 1992.

[20] A. Arneodo, P. Coullet, and C. Tresser, "Possible new strange attractors with spiral structure," Communications in Mathematical Physics, vol. 79, no. 4, pp. 573-579, 1981. 